\title{
Reduction Strategies for Shape Dependent Inverse Problems in Haemodynamics ${ }^{\star}$
}

\author{
Toni Lassila ${ }^{1}$, Andrea Manzoni ${ }^{2}$, and Gianluigi Rozza ${ }^{2}$ \\ 1 EPFL, École Polytechnique Fédérale de Lausanne \\ CMCS, Chair of Modelling and Scientific Computing \\ Station 8, CH-1015, Lausanne, Switzerland \\ 2 SISSA MathLab, International School for Advanced Studies, Trieste, Italy \\ \{toni.lassila, andrea.manzoni, gianluigi.rozza\}@epfl.ch \\ http://cmcs.epfl.ch
}

\begin{abstract}
This work deals with the development and application of reduction strategies for real-time and many query problems arising in fluid dynamics, such as shape optimization, shape registration (reconstruction), and shape parametrization. The proposed strategy is based on the coupling between reduced basis methods for the reduction of computational complexity and suitable shape parametrizations - such as free-form deformations or radial basis functions - for low-dimensional geometrical description. Our focus is on problems arising in haemodynamics: efficient shape parametrization of cardiovascular geometries (e.g. bypass grafts, carotid artery bifurcation, stenosed artery sections) for the rapid blood flow simulation - and related output evaluation - in domains of variable shape (e.g. vessels in presence of growing stenosis) provide an example of a class of problems which can be recast in the real-time or in the many-query context.
\end{abstract}

Keywords: Model order reduction, reduced basis methods, free-form deformation, radial basis functions, computational fluid dynamics, shape parametrization, blood flows.

\section{Introduction and Motivation}

In last decades more and more powerful computers have allowed to solve numerical problems of very large dimensions and describing very complex phenomena. Nevertheless, a computational reduction is still crucial whenever interested to high performances in rapid - even real-time - simulations and/or repeated output evaluations - seen as many queries evaluations- for different values of some inputs of interest.

\footnotetext{
* Work supported by Swiss National Science Foundation (SNSF) under grant 200021122136 and European Research Council under Project Mathcard ERC-2008-AdG227058 .
}

D. Hömberg and F. Tröltzsch (Eds.): CSMO 2011, IFIP AICT 391, pp. 397 406, 2013.

(C) IFIP International Federation for Information Processing 2013 


\subsection{A General Strategy for Reduction in Shape Dependent Flows}

Flow control and optimization problems can be formulated as the minimization of a given cost functional (or output) controlling some input parameters which can be physical quantities (e.g. source terms or boundary values) or, alternatively, geometrical quantities; we refer to the latter case as flow control by shape variation, and the optimization of the corresponding flow geometries is thus one possibility to reach that goal; we refer to this case - the most difficult one among flow control problems - such as shape optimization or shape registration/reconstruction problems [4. Concerning applications arising in fluid mechanics, cost functionals are expressed as functions of flow variables (such as velocity, pressure, temperature), while constraints are usually given in form of PDE systems (Stokes, Navier-Stokes equations, with or without coupling with a structural equation to account for fluid-structure interaction effects) describing the flow, besides topological constraints on the shape of the domain, if necessary. Since (i) optimization procedures require repetitive evaluations of outputs, (ii) PDEs can be hard to solve and (iii) discretization is expensive when geometry keeps changing, computational costs are usually very high; we thus want to address suitable strategies to reduce numerical efforts in many-query problems.

Substantial computational saving becomes possible thanks to a reduced order model which relies on two reduction steps: (i) parameterization of the admissible shapes and (ii) substitution of the full-order finite element (FE) solution of flow problems with a reduced solution obtained by the reduced basis (RB) method [17. In fact, once an equivalent parametrized formulation of the flow problem now embedding the shape as a parametric quantity - can be derived, reduced basis method for parametrized PDEs, enables to evaluate the output very rapidly. In the end, at the outer level a suitable iterative procedure for the optimization is performed. A brief presentation of the whole framework can be found in [8], while a more detailed analysis has been recently addressed in [5]6].

\subsection{Abstract Setting}

From an abstract point of view, a shape optimization/identification can be seen as an optimal control problem for which the control variable is the shape of the domain $\Omega$ itself. This entails the minimization of a cost functional $\mathcal{J}(\cdot)$ over a set of admissible shapes $\mathcal{O}_{a d}$, by finding the optimal shape of the domain where the PDE is defined:

$$
\text { find } \hat{\Omega}=\arg \min _{\Omega \in \mathcal{O}_{a d}} \mathcal{J}(Y(\Omega))
$$

where $\mathcal{J}(Y(\Omega))$ depends on the solution $Y=Y(\Omega)$ of a PDE state problem defined on $\Omega$ - which can be written in an abstract form as

$$
Y \in \mathcal{Y}(\Omega): \quad \mathcal{A}(Y, W ; \Omega)=\mathcal{F}(W ; \Omega), \quad \forall W \in \mathcal{Y}(\Omega) .
$$

Here $\mathcal{A}(\cdot, \cdot ; \Omega)$ is a continuous, uniformly inf-sup stable bilinear form and $\mathcal{F}(\cdot ; \Omega)$ is a bounded linear form, both defined on the original domain $\Omega ; \mathcal{Y}(\Omega)$ denotes a 
suitable functional space defined over $\Omega$. Let us assume that the shape $\Omega=\Omega(\boldsymbol{\mu})$ depends on a set of input parameters $\boldsymbol{\mu}=\left(\mu_{1}, \ldots, \mu_{p}\right) \in \mathcal{D} \subset \mathbb{R}^{p}$; in this way, problem (1)-(2) can be reduced to the following parametric optimization inverse problem:

$$
\text { find } \hat{\boldsymbol{\mu}}=\arg \min _{\boldsymbol{\mu} \in \mathcal{D}_{a d}} \mathcal{J}(Y(\boldsymbol{\mu}))
$$

where $\mathcal{D}_{a d} \subseteq \mathcal{D}$ and $Y(\boldsymbol{\mu})$ solves

$$
Y(\boldsymbol{\mu}) \in \mathcal{Y}(\Omega(\boldsymbol{\mu})): \quad \mathcal{A}(Y(\boldsymbol{\mu}), W ; \boldsymbol{\mu})=\mathcal{F}(W ; \boldsymbol{\mu}), \quad \forall W \in \mathcal{Y}(\Omega(\boldsymbol{\mu})) .
$$

For a more general setting and overview, see e.g. [5].

\section{Reduced Basis Method for Computational Reduction}

Our approach to shape dependent flow problems takes advantage of reduced basis (RB) methods for rapid and reliable prediction of engineering outputs associated with parametric PDEs [1712 14]; see e.g. 15]19]16] for applications to the Stokes problem and 13203] for the Navier-Stokes case. The method is built upon a classical finite element (FE) "truth" approximation space $\mathcal{Y}^{\mathcal{N}}$ of (typically very large) dimension $\mathcal{N}$ and is based on the use of "snapshot" FE solutions of the PDEs, corresponding to certain parameter values, as global approximation basis functions previously computed and stored. The RB framework requires a reference ( $\boldsymbol{\mu}$-independent) domain $\tilde{\Omega}$ in order to compare, and combine, FE solutions that would be otherwise computed on different domains and grids; moreover, this procedure enables to avoid shape deformation and remeshing that normally occur at each step of an iterative optimization procedure [18. In Sect. 3 two possible techniques for the construction of such a mapping will be briefly recalled.

We thus consider $\tilde{\Omega}$ as reference domain related to the parameter-dependent "original" domain of interest $\Omega(\boldsymbol{\mu})$ through a parametric mapping $T(\cdot ; \boldsymbol{\mu})$, s.t. $\Omega(\boldsymbol{\mu})=T(\tilde{\Omega} ; \boldsymbol{\mu})$. By mapping the problem (3) back to the reference domain $\tilde{\Omega}$, we obtain the following problem in its abstract form:

$$
\begin{gathered}
\text { find } \hat{\boldsymbol{\mu}}=\arg \min _{\boldsymbol{\mu} \in \mathcal{D}_{a d}} s(\boldsymbol{\mu})=\tilde{\mathcal{J}}(Y(\boldsymbol{\mu})) \quad \text { s.t. } \\
Y(\boldsymbol{\mu}) \in \mathcal{Y}(\tilde{\Omega}): \tilde{\mathcal{A}}(Y(\boldsymbol{\mu}), W ; \boldsymbol{\mu})=\tilde{\mathcal{F}}(W ; \boldsymbol{\mu}), \quad \forall W \in \mathcal{Y}(\tilde{\Omega}) .
\end{gathered}
$$

Focusing on shape optimization and/or registration problems, and following the so-called discretize than optimize approach, the standard Galerkin FE approximation of (5) reads as follows:

$$
\begin{gathered}
\text { find } \hat{\boldsymbol{\mu}}=\arg \min _{\boldsymbol{\mu} \in \mathcal{D}_{a d}} s^{\mathcal{N}}(\boldsymbol{\mu})=\tilde{\mathcal{J}}\left(Y^{\mathcal{N}}(\boldsymbol{\mu})\right) \text { s.t. } \\
Y^{\mathcal{N}}(\boldsymbol{\mu}) \in \mathcal{Y}^{\mathcal{N}}: \tilde{\mathcal{A}}\left(Y^{\mathcal{N}}(\boldsymbol{\mu}), W ; \boldsymbol{\mu}\right)=\tilde{\mathcal{F}}(W ; \boldsymbol{\mu}), \forall W \in \mathcal{Y}^{\mathcal{N}} .
\end{gathered}
$$

The reduced basis method provides an efficient way to compute an approximation $Y_{N}(\boldsymbol{\mu})$ of $Y^{\mathcal{N}}(\boldsymbol{\mu})$ (and related output) by using a Galerkin projection on 
a reduced subspace made up of well-chosen FE solutions, corresponding to a specific choice $S_{N}=\left\{\boldsymbol{\mu}^{1}, \ldots, \boldsymbol{\mu}^{N}\right\}$ of parameter values. Denoting

$$
\mathcal{Y}_{N}^{\mathcal{N}}=\operatorname{span}\left\{Y^{\mathcal{N}}\left(\boldsymbol{\mu}^{n}\right), n=1, \ldots, N\right\}
$$

the RB space, the RB formulation of (5) is as follows:

$$
\begin{gathered}
\text { find } \hat{\boldsymbol{\mu}}=\arg \min _{\boldsymbol{\mu} \in \mathcal{D}_{a d}} s_{N}(\boldsymbol{\mu})=\tilde{\mathcal{J}}\left(Y_{N}(\boldsymbol{\mu})\right) \text { s.t. } \\
Y_{N}(\boldsymbol{\mu}) \in \mathcal{Y}_{N}^{\mathcal{N}}: \tilde{\mathcal{A}}\left(Y_{N}(\boldsymbol{\mu}), W ; \boldsymbol{\mu}\right)=\tilde{\mathcal{F}}(W ; \boldsymbol{\mu}), \forall W \in \mathcal{Y}_{N}^{\mathcal{N}} .
\end{gathered}
$$

Thanks to the (considerably) reduced dimension $O(N) \ll O(\mathcal{N})$ of the systems obtained from RB approximation, we can provide both reliable results and rapid response in the real-time and multi-query contexts. In particular:

- Reliability is ensured by rigorous a posteriori estimations for the error in the RB approximation w.r.t. truth FE discretization (see e.g. [17/16]);

- Rapid response is achieved by an Offline-Online computational strategy and a rapidly convergent $\mathrm{RB}$ space assembling, based on a greedy algorithm. To achieve this goal, RB methods rely on the assumption of affine parametric dependence 1 in $\mathcal{A}(\cdot, \cdot ; \boldsymbol{\mu})$ and $\mathcal{F}(\cdot ; \boldsymbol{\mu})$.

Hence, in an expensive Offline stage we prepare a very small RB "database", while in the Online stage, for each new $\boldsymbol{\mu} \in \mathcal{D}$, we rapidly evaluate both the field and the output (with error bounds) whose computational complexity is independent of FE dimension $\mathcal{N}$.

\section{Efficient Shape Parametrization Techniques for Geometrical Complexity Reduction}

In general, shape optimization problems feature more difficulties than optimal control problems, such as shape deformation, shape derivatives and the evaluation of shape-dependent quantities: a crucial aspect of optimal shape design is thus the geometrical treatment of the shapes during the optimization process. Common strategies for shape deformation involve the use of $(i)$ the coordinates of the boundary points as design variables (local boundary variation) or (ii) some families of basis shapes combined by means of a set of control point (polynomial boundary parametrizations).

These techniques are not well suited within the RB framework, since a global transformation $T(\cdot ; \boldsymbol{\mu})$ is needed, rather than a boundary representation [18. A more versatile parametrization can be introduced by exploiting the free-form deformation (FFD) techniques, in which the deformations of an initial design, rather than the geometry itself, are parametrized [7]. In this case, the shape

\footnotetext{
${ }^{1}$ If this assumption does not hold, it could be recovered in through an intermediate empirical interpolation process.
} 
parametrization is constructed on a regular lattice of control points, by combining the deformations acting on a subset of active control points through a basis of (tensor products of) Berstein polynomials. Input parameters are given by the deformations of the active control points, which have to be properly chosen, following some problem-dependent criteria [10].

Despite its flexibility, the FFD techniques do not satisfy any interpolation property and control points must reside on a regular lattice. In order to overcome these possible limitations, other different techniques based on interpolation properties may be recovered. In particular, we have been focusing on the radial basis functions (RBF) techniques [9], which are traditionally used for nonlinear multidimensional interpolation on scattered data (for example in image registration). With respect to FFD techniques, RBF techniques allow a better local boundary control and a free choice of the position of the control points (also on the boundary of the shape domain).

\section{Application in Haemodynamics: Real-Time Blood Flow Simulations in Parametrized Cardiovascular Geometries}

The framework based on the coupling between FFD or RBF techniques (or other low-dimensional shape parametrizations) and RB methods has turned out to be useful also for a real-time simulation of blood flows in arterial vessels which might show a deep variation in geometrical configuration, as for example carotid artery bifurcations. Our goal is twofold:

- spanning a variety of carotid configurations through low-dimensional shape parametrizations [1], and shape registration of parametrized carotid shapes from patient data measured in the form of flow velocities;

- real-time simulation of blood flows in reconstructed geometries and computing indices related to arterial occlusion risk and highly dependent on geometrical configurations, possibly for predictive surgery applications.

In the first approach we might minimize some discrepancy functional between the simulated velocity and the observed velocity in an atlas-based variational data assimilation method (see e.g. [11]); in the latter we minimize a cost function such as the viscous energy dissipation

$$
\mathcal{J}(Y(\boldsymbol{\mu}))=\frac{\nu}{2} \int_{\Omega}|\nabla \mathbf{u}(\boldsymbol{\mu})|^{2} d \Omega .
$$

to obtain carotid shapes exhibiting the least disturbance to the blood flow, being $Y(\boldsymbol{\mu})=(\mathbf{u}(\boldsymbol{\mu}), p(\boldsymbol{\mu}))$ the velocity and the pressure of the fluid, respectively.

\subsection{Validation of the Reduced Basis Methodology}

A first numerical test has been performed exploiting a coupled FFD+RB framework on a simple geometrical configuration (see Fig. 1), given by a stenosed 
carotid artery parametrized with respect to the displacement of two control points $(p=2)$ located close to the bifurcation (see e.g. 9] for further details about representation of carotid bifurcations). Flow simulations through a steady Stokes model show a remarkable dependence of the flow even on small variation of the shape configuration. In particular, our interest has been focused on the evaluation of an output related both with the flow and the shape, given by the viscous energy dissipation.

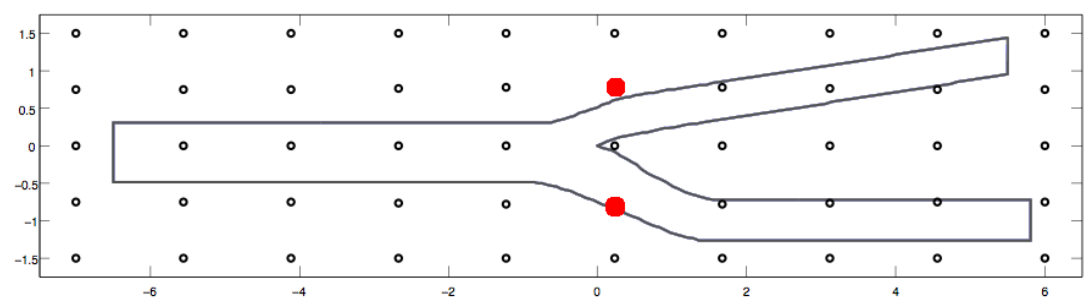

Fig. 1. Schematic diagram of the FFD setting; bold (red) control points can be freely moved in vertical direction and used as parameters representing small deformations

Some details concerning the reduced basis spaces are listed in Tab. 1; we remark the strong reduction in the system dimensions and a large computational speedup, concerning performances for each new geometrical configuration, of about two orders of magnitude. We provide a certification of the accuracy of the methodology: in Fig. 2 the true errors between the FE and the RB approximation are reported, the related error bounds (see [16] for error bound expression and derivation), as well as the error between the FE and the RB output. We observe fast, nearly exponential convergence in $N$. Furthermore, the a posteriori error bounds are both reliable and reasonably effective.

Table 1. RB + FFD for the carotid artery bifurcation: numerical details

\begin{tabular}{lc}
\hline Number of FE dof $\mathcal{N}_{\mathbf{v}}+\mathcal{N}_{p}$ & 24046 \\
\hline Number of RB functions $N$ & 16 \\
Number of design variables $P$ & 2 \\
\hline Linear system dimension reduction & $500: 1$ \\
FE evaluation $t_{F E}^{\text {online }}(\mathrm{s})$ & 2.8039 \\
RB evaluation $t_{R B}^{\text {online }}(\mathrm{s})$ & 0.0231 \\
\hline
\end{tabular}

\subsection{A Comparison between FFD and RBF Parametrizations}

Next, we report here some preliminary results on the comparison between a FFD and a RBF setting defined on the carotid configuration already introduced. Also in this case we are interested in the evaluation of the viscous energy dissipation; 

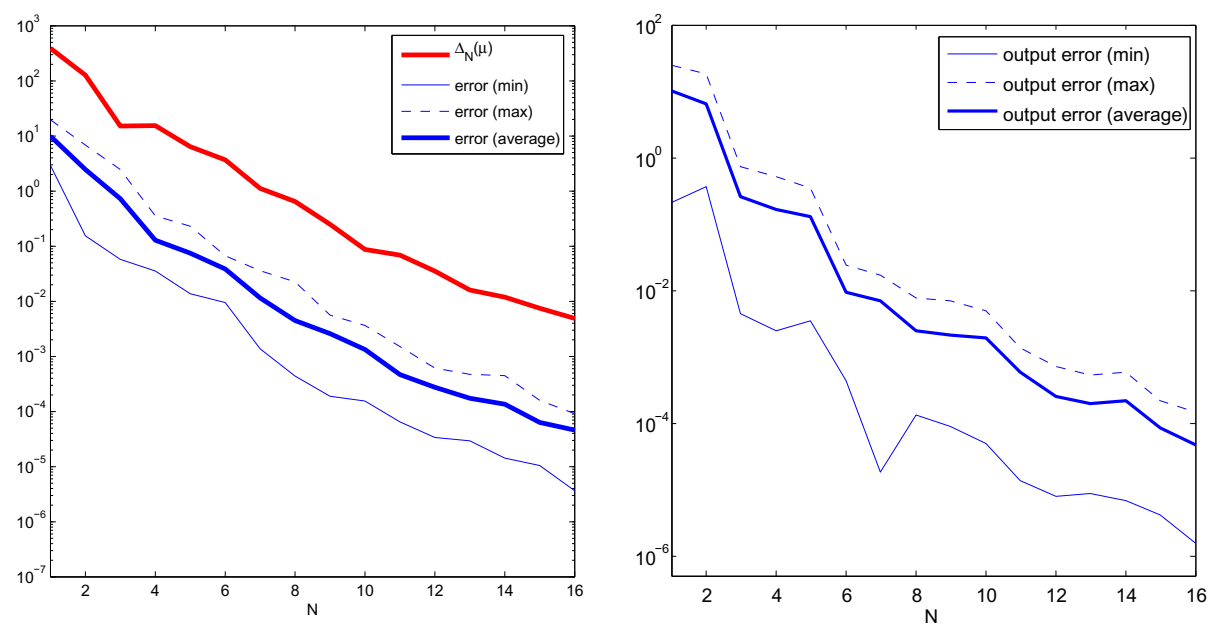

Fig. 2. Left: • error estimation (natural norm) and • true error between RB and FE approximation; right: true error between FE and RB output (vorticity)
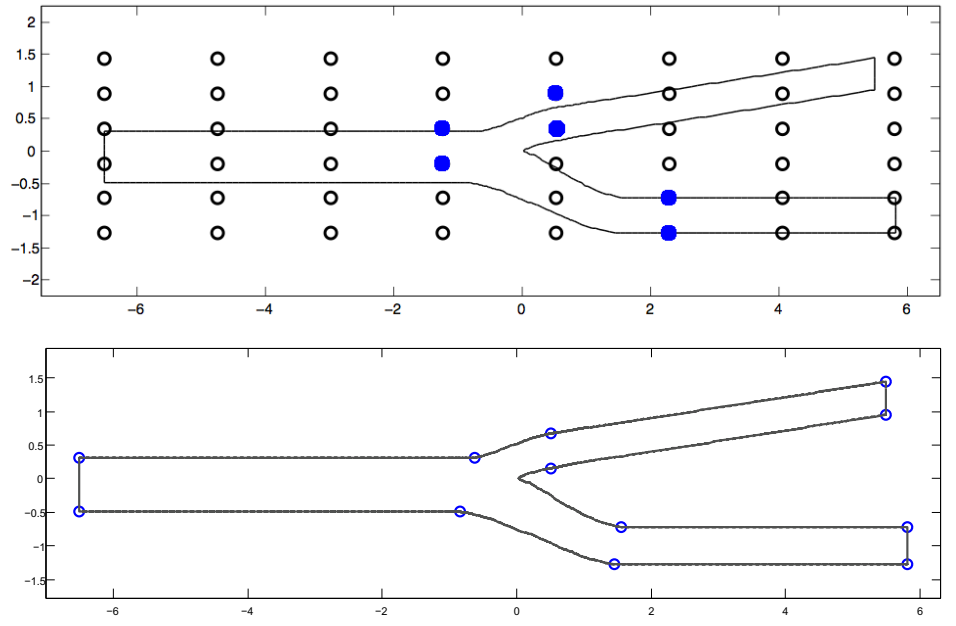

Fig. 3. FFD setting (top) and RBF setting (bottom); for each case, parameters are given by the displacements of the selected (blue) control points

we just compare the two parametrizations techniques by considering a NavierStokes model for the fluid flow. Both the settings deal with $p=6$ parameters, given by the vertical displacements of some selected control points; in the FFD case we introduce a $6 \times 8$ lattice of control points, while in the RBF case we introduce in total 12 control points close to the bifurcation and at the extrema (see Fig. 3), using the thin-plate spline (TPS) and the Gaussian shape functions 
Table 2. Results for the minimization of the viscous energy dissipation obtained by using the FFD and the RBF settings introduced above

\begin{tabular}{rccc}
\hline & FFD & RBF (thin-plate) RBF (Gaussian) \\
\hline output reduction & $39,1 \%$ & $45,9 \%$ & $36,7 \%$ \\
\hline iterations & 84 & 117 & 91 \\
parameters & $6(48)$ & $6(12)$ & $6(12)$ \\
\hline
\end{tabular}
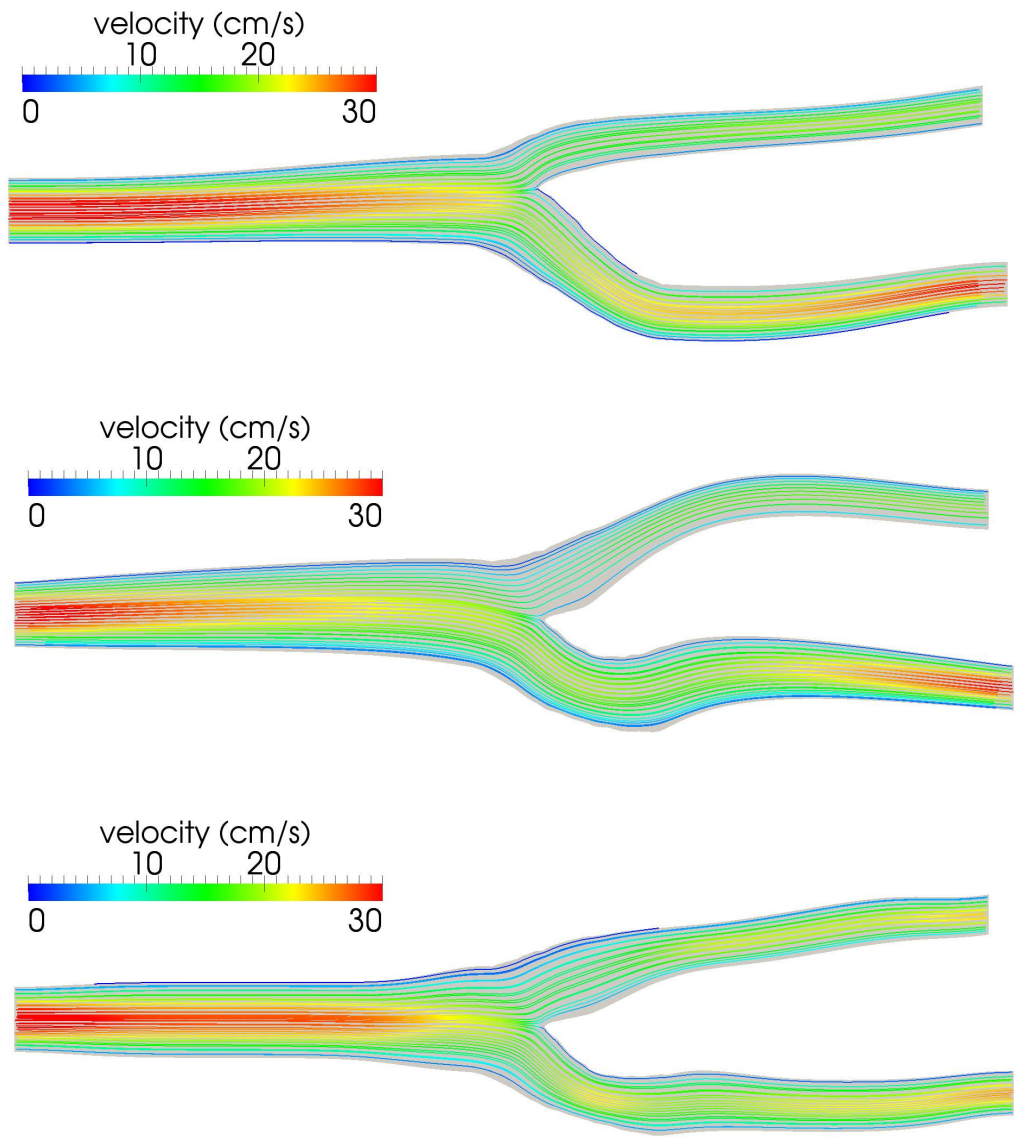

Fig. 4. Optimal configuration obtained by minimizing the viscous energy dissipation for the FFD case (top), the RBF case with the thin-plate spline option (middle) and the RBF case with the Gaussian option (bottom)

2. In this last case, we deal with the displacement of the six control points located at the center of the configuration.

We compare the shapes obtained by minimizing the viscous energy dissipation: in Tab. 2 are reported the results for the two cases, while the configurations 
corresponding to the minimum values of the viscous energy dissipation are represented in Fig. 4. We can remark that the three shapes are quite similar, as well as the output reduction. The number of iterations taken by the optimization procedure is comparable among the three options. Regarding the main qualities of these two shape parametrization tools, the RBF technique proves to be more versatile and accurate for this kind of applications - it enables to choose freely the location of control points rather than selecting the most relevant control points on the regular FFD lattice, as well as to impose interpolation constraints - even if its construction and the computation of the related parametrized tensors is much more difficult. Not only, by considering the same amount, location and available displacements of control points, the Gaussian RBF is found to be more suitable for describing local deformations; the TPS option allows to get a more global and regular deformation, where an enhanced shape smoothness is ensured by a minimization of the bending deformation energy property, fullfilled by this kind of RBF.

\section{Conclusion and Perspectives}

The capability of the reduced basis method to solve shape registration and optimization problems involving incompressible flows in real-time looks promising if coupled with an efficient and versatile geometrical parametrization. The integration of the RBF parametrization technique within the reduced basis framework, as well as its application to blood flow simulation on geometries reconstructed from patient data, looks promising in its flexibility and ability to express a variety of shape deformations. Further elements that may be explored deal with the uncertainty quantification [5] and/or robust optimization and control problems [6] for patient-specific scenarios.

\section{References}

1. Bressloff, N.W.: Parametric geometry exploration of the human carotid artery bifurcation. J. Biomech. 40, 2483-2491 (2007)

2. Buhmann, M.D.: Radial Basis Functions. Cambridge University Press, UK (2003)

3. Deparis, S., Rozza, G.: Reduced basis method for multi-parameter-dependent steady Navier-Stokes equations: Applications to natural convection in a cavity. J. Comp. Phys. 228(12), 4359-4378 (2009)

4. Haslinger, J., Mäkinen, R.A.E.: Introduction to shape optimization: theory, approximation, and computation. SIAM (2003)

5. Lassila, T., Manzoni, A., Quarteroni, A., Rozza, G.: A reduced computational and geometrical framework for inverse problems in haemodynamics (2011) (submitted)

6. Lassila, T., Manzoni, A., Quarteroni, A., Rozza, G.: Boundary control and shape optimization for the robust design of bypass anastomoses under uncertainty. Accepted for publication in ESAIM Math. Model. Numer. Anal. (2012), doi: $10.1051 / \mathrm{m} 2 \mathrm{an} / 2012059$

7. Manzoni, A., Quarteroni, A., Rozza, G.: Shape optimization for viscous flows by reduced basis methods and free-form deformation. Int. J. Numer. Meth. Fluids 70(5), 646-670 (2012) 
8. Manzoni, A.: Model order reduction by reduced basis for optimal control and shape optimization. Paper Awarded with the 3rd BGCE Student Paper Prize at the 2011 SIAM Computational Science and Engineering Conference, Reno, NV, USA (2011)

9. Manzoni, A., Quarteroni, A., Rozza, G.: Model reduction techniques for fast blood flow simulation in parametrized geometries. Int. J. Numer. Methods Biomed. Engng. (2011), doi:10.1002/cnm.1465) (in press)

10. Manzoni, A., Quarteroni, A., Rozza, G.: Shape optimization of cardiovascular geometries by reduced basis methods and free-form deformation techniques. Int. J. Numer. Methods Fluids (2011), doi:10.1002/fld.2712) (in press)

11. McLeod, K., Caiazzo, A., Fernández, M., Mansi, T., Vignon-Clementel, I., Sermesant, M., Pennec, X., Boudjemline, Y., Gerbeau, J.F.: Atlas-based reduced models of blood flows for fast patient-specific simulations. Statistical Atlases and Computational Models of the Heart, 95-104 (2010)

12. Patera, A.T., Rozza, G.: Reduced Basis Approximation and A Posteriori Error Estimation for Parametrized Partial Differential Equations. Version 1.0, Copyright MIT, to appear in (tentative rubric) MIT Pappalardo Graduate Monographs in Mechanical Engineering (2006), http://augustine.mit.edu

13. Quarteroni, A., Rozza, G.: Numerical solution of parametrized Navier-Stokes equations by reduced basis methods. Numer. Methods Partial Differential Equations 23(4), 923-948 (2007)

14. Quarteroni, A., Rozza, G., Manzoni, A.: Certified reduced basis approximation for parametrized partial differential equations in industrial applications. J. Math. Ind. 1(3) (2011)

15. Rozza, G.: Reduced basis methods for Stokes equations in domains with non-affine parameter dependence. Comput. Vis. Sci. 12(1), 23-35 (2009)

16. Rozza, G., Huynh, D.B.P., Manzoni, A.: Reduced basis approximation and error bounds for Stokes flows in parametrized geometries: roles of the inf-sup stability constants. Numer. Math (in press, 2013)

17. Rozza, G., Huynh, D.B.P., Patera, A.T.: Reduced basis approximation and a posteriori error estimation for affinely parametrized elliptic coercive partial differential equations. Arch. Comput. Methods Engrg. 15, 229-275 (2008)

18. Rozza, G., Manzoni, A.: Model order reduction by geometrical parametrization for shape optimization in computational fluid dynamics. In: Pereira, J.C.F., Sequeira, A. (eds.) Proceedings of ECCOMAS CFD 2010, V European Conference on Computational Fluid Dynamics, Lisbon, Portugal (2010)

19. Rozza, G., Veroy, K.: On the stability of the reduced basis method for Stokes equations in parametrized domains. Comput. Methods Appl. Mech. Engr. 196(7), 1244-1260 (2007)

20. Veroy, K., Patera, A.T.: Certified real-time solution of the parametrized steady incompressible Navier-Stokes equations: rigorous reduced-basis a posteriori error bounds. Int. J. Numer. Methods Fluids 47, 773-788 (2005) 